\title{
Work study of a desk computer in the clinical laboratory
}

\author{
J. G. LINES \\ From the Biochemical Section, John Bonnett Clinical Laboratories, \\ Addenbrooke's Hospital, Cambridge
}

SYNOPSIS Calculating results from laboratory data is a time-consuming part of laboratory work, particularly in chemical pathology, and is a frequent source of mistakes. It is much less popular with technical staff than the basic laboratory work.

The reasons for purchasing a desk computer, and its use to perform the calculations in a number of investigations and to convert AutoAnalyzer data into concentration units, are discussed.

The introduction of a desk computer improved morale, speeded up the work, and reduced both fatigue and mistakes.

The need for and the advantages of automating data handling are now well recognized in clinical pathology, and methods of facilitating the handling of requests and reporting results have been considered recently by a Working Party of the Association of Clinical Pathologists (1968). The computation of the test results from the basic analytical data was, however, not discussed. This is a problem, particularly in the chemical pathology laboratory, but to a lesser extent it is also present in the other branches of pathology. Basic analytical data in the chemistry laboratory are mostly colorimeter readings in one form or another, which, by reference to standards, have to be converted into concentration units. Sometimes, further calculations have to be made, for example, to determine 24-hour excretion rates. Other less frequently performed computations with the basic data, such as the Allen or Talbot corrections of optical density readings in steroid analyses, are more demanding in time and ability, and statistical analysis of the final data is necessary for adequate quality control.

All such calculations involve only elementary arithmetical procedures, but are tedious when done on the scale nowadays necessary in the average size hospital laboratory where sophisticated computer facilities are unlikely to be available. If it would be possible to program a calculating machine, these calculations could be automated, thereby saving time and reducing both fatigue and the number of mistakes. The purpose of this communication is to state the reasons for purchasing an Olivetti

Received for publication 31 October 1968
Programma 101 desk computer ${ }^{1}$ and to exemplify and evaluate its use.

The present annual workload of our laboratory is 135,000 tests. Of these, some 90,000 are plasma sodium, potassium, and bicarbonate, and urea and blood sugar determinations, all of which are performed on AutoAnalyzers. The translation of the basic AutoAnalyzer data into the final values for reporting not only occupied more than 200 technician hours per annum, but more importantly, was the source of an increasing number of mistakes. It was possible to relate the incidence of these mistakes to the increasing pressure of work, particularly on the less experienced technical staff. Requests for the arithmetically more demanding investigations, for example, urinary 17 oxo- and oxogenic steroids, creatinine clearances, and urine oestriol assays, currently average 140,130 , and 40 per month, respectively. The calculations from these investigations alone, when performed without the aid of a calculator, require 135 to 160 technician hours per year and constitute the most disliked part of the work. As the number of all types of investigations can be expected to continue increasing in the future, it is imperative to automate data computations wherever possible.

It was not possible for the laboratory to share on-line or off-line computer facilities in the foreseeable future and the cost of setting up such facilities independently was prohibitive. The Olivetti Programma 101 (Fig. 1), however, appeared to suit our requirements. It had printout facilities which we

${ }^{1}$ British Olivetti Ltd, 278 High Holborn, London. 


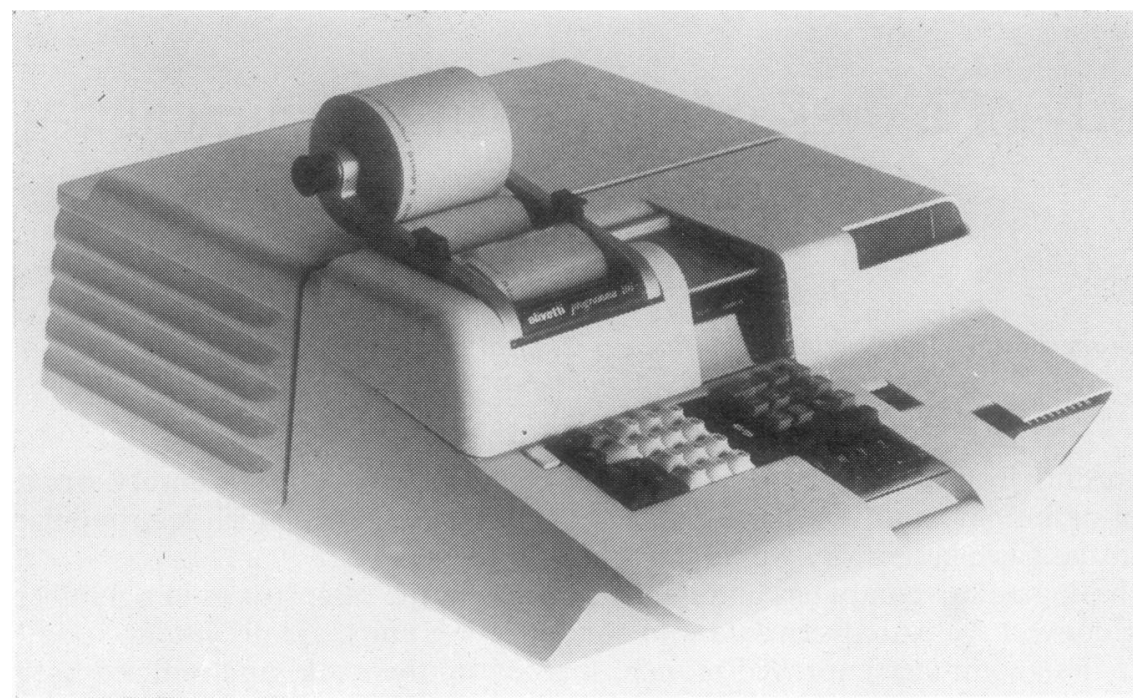

FIG. 1. The Olivetti Programma 101 desk computer (dimensions $24 \times 19 \times 7 \frac{1}{2}$ in.).

considered obligatory for verifying work, and, while having the simple keyboard of the conventional calculating machine, could be programmed by means of magnetic cards. The machine could, therefore, store instructions and data, and carry out a sequence of instructions so that for any computation only the variable numerals had to be tabulated into the machine. Both the need for operators to learn the variety of manipulations necessary with conventional calculators and the possibility of subsequent mistakes were eliminated. Its programming capacity of 120 instructions and up to 10 storage registers is adequate for reading AutoAnalyzer standard curves. Furthermore, its $\cos ^{2}$ is not excessive for the average size laboratory and is considerably less than that of other calculators with the same facilities.

\section{LABORATORY APPLICATIONS OF PROGRAMMA 101}

Introducing the Programma 101 into the laboratory necessitated only small changes in the usual work pattern. For reading AutoAnalyzer curves, the system found most convenient is that, as each standard curve becomes available at the beginning of the day, to insert the curve analysis program card into the machine and tabulate the values for each standard into separate registers, and then to re-record the program and data onto the appropriate magnetic card, ie, potassium standard curve, bicarbonate standard curve, etc, for storage until required. This entire procedure takes about one and a half minutes and all cards can be used day after day.

${ }^{2}$ Present cost $£ 1,720$.
Concentration units for any peak height or batch of peak heights can be obtained when required by inserting the appropriate card, pressing the pro- 0 gram selection button, and tabulating in the peak height values. The printout records the peak heights followed by the concentration units. The curve reading program first determines between which pair of standard values the test peak height lies, and then calculates the test concentration units from the regression equation relating the two standard values.

Small alterations in shape or height of the standard curves, inherent in many AutoAnalyzer procedures: during extended use, must be taken into account when calculating the concentration of the test solu= tion. One of the easiest methods of making $a \bar{p}$ correction for drift, and in our experience the most. valid practical procedure, is to make a proportiona $\underline{B}$ correction to each sample peak height, for the shifk of peak height of the standard at that part of the standard curve which is most affected, usually in the region of low absorbance. Although this procedure would be prohibitively time consuming without auto+N mated calculation facilities, the Olivetti Programma 101 performs the calculation in about 0.2 seconds $N$ and the instructions for this procedure can bet incorporated into the curve reading program. The complete program contains four parts. In the first of these, the factor by which the standard peak heigh differs from its original value is calculated and? stored. Then this factor is used to correct each sub-0 sequently entered peak height before entering tha $\bar{P}_{\vec{P}}$ peak height to the curve reading and calculation parts of the program. Drift standards can be use $\frac{10}{2}$ 
as often as necessary, usually about every sixth to tenth sample. It takes approximately five seconds, with or without drift correction, to determine the concentration of any sample from its peak height. Of this time, approximately three seconds are available to examine the next peak and decide upon its value. Although the total working time in computing AutoAnalyzer results is not significantly different from the conventional manual methods, the extended peak examination time minimizes peak reading errors and the fatigue associated with reading curves visually is virtually eliminated.

In those investigations which require more involved mathematical treatment of the basic data than transformation to other units, not only are fatigue and mistakes reduced, especially when decimals are involved, but saving time becomes significant. Calculating a batch of 12 creatinine clearance values from their blood and urine concentrations and the urine volume collected in the timed period takes just over nine minutes using a slide rule. A program has been written for the Programma 101 to calculate clearances and the same 12 calculations take 2.3 minutes. The actual saving in time is, however, somewhat greater because whereas the previous practice had been to check manual calculations by a second more senior worker repeating them, it is now only essential to check the validity of the basic data inserted into the machine. On a workload of about 130 such batches annually, the total time saved is more than 35 technician hours.

With analyses involving calculations which are more difficult by slide rule than those of creatinine clearance, saving time becomes proportionately greater. Urine steroid analyses require the Talbot correction (Talbot, Berman, and MacLachlan, 1942) for the optical densities of test and standard solutions at $520 \mathrm{~nm}$. The calculation below usually takes 40 seconds

$$
\text { Corrected } \mathrm{OD}_{520}=\frac{\mathrm{OD}_{520}-\left(0.6 \times \mathrm{OD}_{430}\right)}{0.73}
$$

and then a further 35 to 50 seconds is required to calculate the 24-hour excretion value. Experienced workers need 55 to 70 minutes to calculate and check the results of a batch of 20 urine steroid results by hand, whereas the same results are obtained in six minutes using the Programma. Analysing 75 such batches a year, the total time saved is about 80 technician hours per year. Similar investigations of urine oestriol excretion calculations incorporating the Allen (1950) correction show an estimated annual gain of 22 hours in technician time on an annual workload of 500 analyses.
For the simplest of laboratory calculations, such as those of the straightforward colorimetric procedures in which the optical density of a number of test solutions is related to the standard value and concentration, the amount of time saved using the desk computer is comparatively small. However, its use for such calculations, and those of other similar analyses, such as enzyme assays which necessitate the additional preliminary calculation of subtracting blank or control values from each test value, does reduce fatigue and mistakes. Quality control procedures, eg daily means, are facilitated by the use of the desk computer. To obtain the mean of 120 values takes approximately two minutes, and if the standard deviation about that mean is required, this takes a few seconds longer.

\section{PROGRAMMING}

Programs such as those already referred to are not difficult to write. The keyboard is similar to that of a conventional calculating machine and the Programma can be used in the manual mode. When used manually, not only do the figures which are tabulated into the machine appear on the paper printout, but also symbols corresponding to the manipulations which were performed with the variables and the keys which were pressed to initiate the manipulations. To program the machine for the same calculation, it is only necessary to depress a button designated 'record program' and then to repeat the sequence of operations in the example by following the symbols on the sample printout, but depressing key $\mathbf{S}$ instead of each variable, and preceding and ending the program with 'beginning of program' and 'recommence program' instructions. Any constants involved can be tabulated into the storage registers. The program can then be stored permanently on a magnetic card by passing a card through the read/write head. More widely applied programs, such as those for statistical calculations, rarely need to be devised as they are available in a program manual supplied free by the manufacturers. The principles of including subroutines, internal loops, etc, in programs can be learnt from the instruction booklets accompanying the machine, but for those who desire it, programming instruction courses are provided by the manufacturers.

\section{CONCLUSIONS}

At the time of writing, a Programma 101 has been in use for just over six months. Even after this relatively short time it is possible to draw a number of conclusions. That aspect of work which techni- 
cians dislike most, namely, calculations, has been largely eliminated and consequently morale has improved. An 80 to $90 \%$ saving in time at the calculation stage of a number of laboratory procedures has been achieved and should result in about 200 technician hours annually being available for additional analytical work. The greatest benefit, however, is the increased reliability of results, directly through the minimizing of errors in calculation and indirectly through statistical control procedures which the machine facilitates. In AutoAnalyzer work, improved laboratory accuracy can be achieved mainly through the opportunity to examine peaks more carefully without any reduction in work output. While technicians will normally take one second or less to examine a peak, there is no incentive to hurry when using the Programma because the keyboard is locked for the five seconds during which the computation takes place, and the incidence of reading errors is consequently reduced.
The Programma 101 has proved more valuable $\frac{\text { o }}{-}$ than had been originally anticipated. Not only can $\overrightarrow{\vec{F}}$ it be used as a programmed calculator for repetitive work, but it can be used in the manual mode for $\frac{}{\circ}$ single calculations. However, when more than a few $\overline{\bar{c}}$ calculations are required, it is advantageous to write $\overrightarrow{\mathbb{D}}$ a program. For most procedures this presents little $\stackrel{\circ}{0}$ difficulty and for this reason its applications and ${ }^{\infty}$ utilization should increase. For the average size $\overrightarrow{0}$ laboratory in which sophisticated computer facilities $\vec{A}$ are unlikely to be available for some time, the $\tilde{O}^{-}$ computerized desk calculator appears to be a useful acquisition.

\section{REFERENCES}

Allen, W. M. (1950). J. clin. Endocrinol., 10, 71. port, Data Processing in Clinical Pathology. J. clin. Path., 21, 231.

Talbot, N. B., Berman, R. A., and MacLachlan, E. A. (1942). J. biol. Chem., 143, 211.

\section{Reports and Bulletins prepared by the Association of Clinical Biochemists}

The following reports and bulletins are published by the Association of Clinical Biochemists. They may be obtained $\vec{P}$ from The Administrative Office, Association of Clinical Biochemists, 7 Warwick Court, Holborn, London, W.C.1. The prices include postage, but airmail will be charged extra. Overseas readers should remit by British Postal or Money Order. If this is not possible, the equivalent of $10 \mathrm{~s}$. is the minimum amount that can be accepted.

\section{SCIENTIFIC REPORTS}

3 Automatic Dispensing Pipettes. An assessment of 35 commercial instruments. 1967. P. M. G. BROUGHTON, A. H. GOWENLOCK, G. M. WIDDOWSON, and K. A. AHLQUIST. 10s.

\section{TECHNICAL BULLETINS}

9 Determination of Urea by AutoAnalyzer. November 1966. RUTH M. HASLAM. 2s. 6 d.

11 Determination of Serum Albumin by AutoAnalyzer using Bromocresol Green. October 1967. B. E. NORTHAM and G. M. WIDDOWSON. 2s. 6d.

12 Control Solutions for Clinical Biochemistry. February 1968. P. M. G. BROUGHTON. 2s. 6 d.
13 An Assessment of the Technicon Type II Sampler Unit. March 1968. B. C. GRAY and G. K. MCGOWAN. 1s. 6d.

14 Atomic Absorption Spectroscopy. An Outline of its Principles and a Guide to the Selection of Instru-o ments. May 1968. J. B. DAwson and P. M. G. BROUGHTON. 4s.

15 A Guide to Automatic Pipettes (2nd edition). June N 1968. P. M. G. BROUGHTON. 5s.

16 A Guide to Automation in Clinical Chemistry. May? 1969. P. M. G. BROUGHTON 12s. 6d. (\$1.50.)

17 Flame Photometers. P. WILDING. (To be published soon.) 